\title{
Monocular pose measurement method based on circle and line features
}

\author{
Cai Meng ${ }^{1, a}{ }^{,}$Hongchao Sun ${ }^{1, b}$ \\ ${ }^{1}$ BeiHang University ,China Beijing 100191 \\ aTsai@buaa.edu.cn \\ bzy1415212@buaa.edu.cn
}

Keywords: monocular vision; position-posture determination; circle; line.

\begin{abstract}
In this paper, a new method based on circle and linear projection is proposed. In a straight line and a circle on the target space as the main object recognition. In the case of knowing the radius of the space circle and coordinate of a point on the space line: First of all, we can use the space circle projection to solve all possible solutions, there are two groups. Secondly, determine the rotation angle of space circle around normal vector in the two groups by using the relative position relation of space straight line and circle. Then, we can get the rotation relation between the object coordinate system and the camera coordinate system and get the coordinate representation of circle and straight line in the camera coordinate system in two groups of solutions. Finally, project the point on the space line onto the image plane in two groups of solutions. In the correct solution, the distance between the projection point and the projection line is small. The experimental results show that the method can find the correct solution in all solutions and determine the rotation angle of the space circle around the normal vector. The method can get the correct result under the condition of noise, so the method has strong robustness.
\end{abstract}

\section{Introduction}

Relative pose measurement is of great significance to the near distance rendezvous and docking of the orbit spacecraft and the target acquisition of space robot arm [1-3]. Image based vision pose measurement technology has high precision, good adaptability, and it is the main method to realize the relative position and posture measurement [4-6].

Docking ring is a common part of the aircraft on orbit docking function, and its projection on the image is generally oval. The ellipse is relatively stable on the image with different distance, so it becomes a new method to measure the relative position and posture by using the projection ellipse on the image of the butt joint ring [7,8,12].

Shiu[9] proposed to use single circle structure corresponding to the elliptic eigenvalue pose settlement method, the computational results are ambiguous, and only you can determine the direction of vector method of space circle, unable to determine spatial circle around the rotating angle of the vector method. Zhang [10] and so on by the addition of laser range finder, to solve the problem of the Shiu results in the two sense, but the method is still unable to determine the angle of the circle around the law vector. Safaeerad[11] on multiple vector method in the same space circle imaging, and then use the parallel vector method of constraint resolve the problem of ambiguity in the understanding, but this method requires at least two round, when the scene space cannot provide enough feature, this method cannot be used. Seedlings [12] the space circular structure point to Euclidean invariance from the center of the circle as the constraint conditions, to solve the problem of ambiguity, but can't determine the spatial circle around the rotating angle of the vector method.

In summary, the existing problems in the existing solutions are mainly: the results have two meanings, the posture results can't determine the angle of the circle around the normal vector.

In view of the above problems, this paper presents the solution of the position and posture of the circular feature and the linear feature of the aircraft.

First, using the projection circle to determine the spatial circle of position and plane normal vector, due to the presence of ambiguous, so there are two sets of solutions. 
Secondly, the relative position relation between straight line and space circle is used to determine the angle of rotation of the space circle around the normal vector in the two groups. And then the rotation transformation between the space coordinate system and the camera coordinate system is obtained.

Finally, the point on the space line project onto the image plane in two groups (the rotation transformation and translation transform are obtained). In the correct solution, the projection point to the projection line is small.

The experimental results show that this method can solve the problem of the two meaning of the position and posture solution, and determine the rotation angle of the space circle around the normal vector, and get the result of the full pose. Ellipse feature and straight line feature are both set features, and they have higher stability with respect to the point feature, so the method proposed in this paper has higher robustness and stability.

\section{Position and Pose Calculation Model}

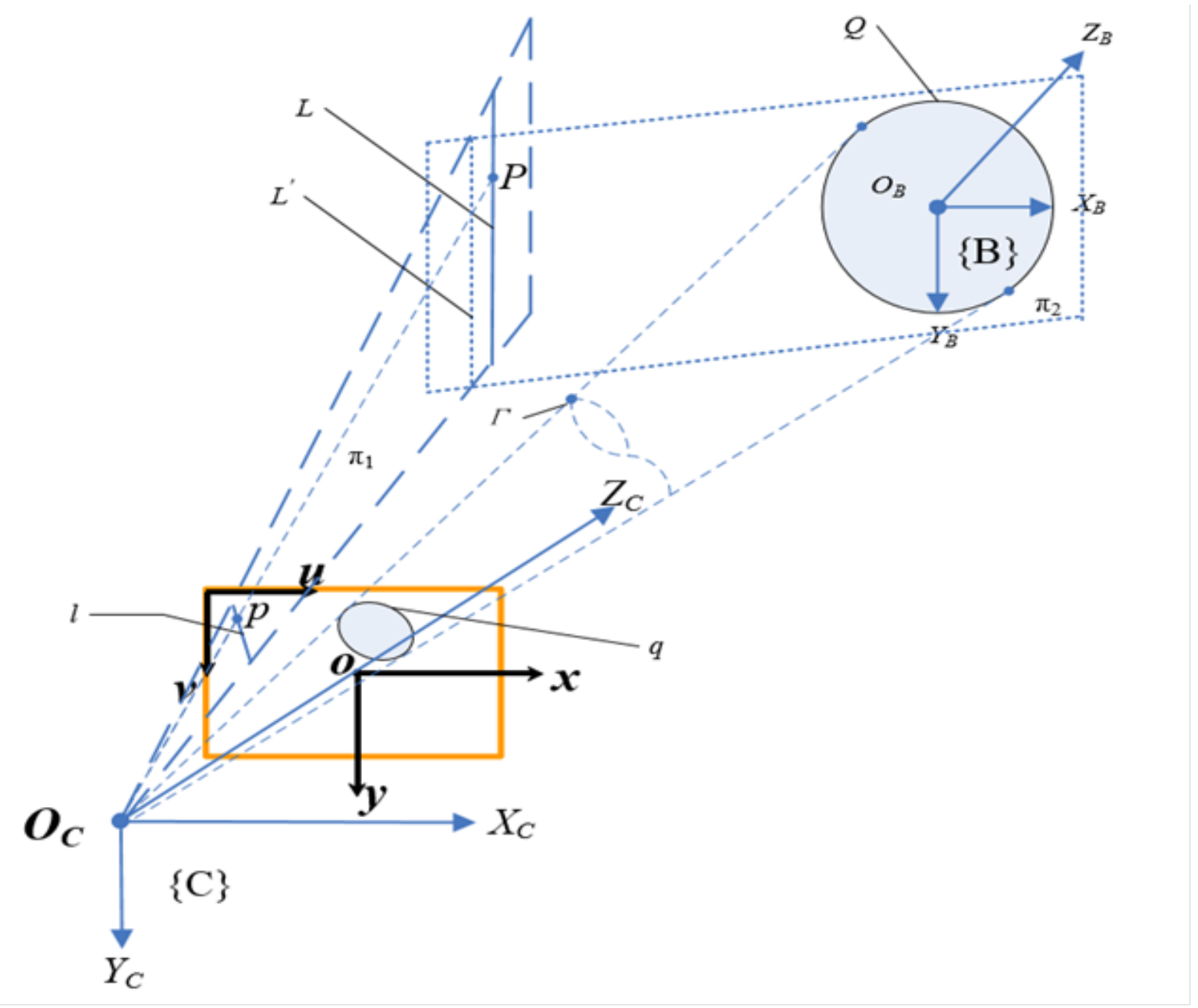

Fig. 1 Position and posture calculation model

Fig. 1 shows pose determination model via the 3D circle and line projection. In Fig. $1\{\mathrm{C}\}$ is the camera reference frame in which $\mathrm{Oc}_{c} \mathrm{XcY} c \mathrm{Zc}$ is the coordinate. Oc is the center of camera lens, and $O c Z c$ is the optical axis pointed forward of the camera. I is the image plane of the camera, $u-v$ is the pixel coordinate. $o$ - $x y$ is the physical coordinate of the image plane.

$Q$ is the circle on the object, and $\pi 2$ is the supporting plane of $Q . O b$ is the center of the circle. $O b Z b$ is the normal of $\pi 2$, and the angle between $O b Z b$ and $O c Z c$ is acute. Let $\{\mathrm{B}\}$ be local reference 
frame of the object, and $O b-X b Y b Z b$ is the local coordinate. $L$ is a space line on the object and is parallel to $O b X b$.

$Q$ is the image of the circle $Q$ on the image plane $I$, and $l$ is the image of the line $L$. Normally, $q$ is an ellipse while $l$ is still a line. We ignore the special case that $q$ becomes a line or $l$ becomes a point. $\pi 1$ is a plane determined by $L$ and $O b$ and $\Gamma$ is an elliptical cone determined by $q$ and $O c$.

2.1 Preliminary calculation

As known, with monocular image of a known circle in a calibrated camera, its center position and normal direction of supporting plane can be acquired. For clarification, we introduce briefly here. Suppose ellipse q in image I have been extracted, and its equation is,

$a u^{2}+b v^{2}+c u v+d u+e v+f=0$

where $(a, b, c, d, e, f)$ are fitted parameters of the extracted ellipse $q$.

Rewrite Eq.(1) into algebra form,

$\left[\begin{array}{lll}u & v & 1\end{array}\right] C\left[\begin{array}{lll}u & v & 1\end{array}\right]^{T}=0$

where, $C q$ is the quadratic form of ellipse $q$, and

$$
C=\left[\begin{array}{ccc}
a & c / 2 & d / 2 \\
c / 2 & b & e / 2 \\
d / 2 & e / 2 & f
\end{array}\right]
$$

For any 3D point $P$, suppose $P\{C\}=\left[\begin{array}{lll}x & y & z\end{array}\right]$ be its coordinates in reference frame $\{C\}$, and $p=[u, v]$ is its image in $I$, then,

$$
z\left[\begin{array}{l}
u \\
v \\
1
\end{array}\right]=\left[\begin{array}{cccc}
f_{u} & 0 & u_{0} & 0 \\
0 & f_{v} & v_{0} & 0 \\
1 & 1 & 1 & 0
\end{array}\right]\left[\begin{array}{l}
x \\
y \\
z \\
1
\end{array}\right]=K\left[\begin{array}{l}
x \\
y \\
z \\
1
\end{array}\right]
$$

$(x, y, z)$ is Camera coordinate system, $(\mathrm{u} 0, \mathrm{v} 0)$ is camera principal point, in pi, $(\mathrm{fu}, \mathrm{fv})$ is the camera's focal ratio, $K$ is the intrinsic parameter matrix of the camera.

Substitute Eq.(4) into Eq.(2), Eq.(2) can be rewritten as,

$$
\left[\begin{array}{lll}
x & y & z
\end{array}\right] K^{T} C K\left[\begin{array}{lll}
x & y & z
\end{array}\right]^{T}=0
$$

Let

$$
Q_{\Gamma}=K^{T} C_{\Gamma} K
$$

And $Q \Gamma$ is the quadric form of elliptic cone $\Gamma$ in reference frame $\{C\}$ with the origin $O c$ being the conical tip. In $\{C\}, Q \Gamma$ is complex since the axis of $\Gamma$ is not coincided with the coordinate axis. Suppose the axis of $\Gamma$ be $O c Z^{\prime}$, and $O c X^{\prime}$ is parallel to the long axis of sectional ellipse of $\Gamma$. Then in coordinate $O c-X^{\prime} Y^{\prime} Z^{\prime}, \Gamma$ becomes standard elliptic cone. Since the origins of $O c-X c Y c Z c$ and $O c$ $-X^{\prime} Y^{\prime} Z^{\prime}$ are same, there must be a pure rotation transformation between them. Obviously, $Q \Gamma$ is a symmetric matrix, then there must be an orthogonal matrix $P$ s.t.,

$$
P^{T} Q P=P^{-1} Q P=\operatorname{diag}\left\{\lambda_{1}, \lambda_{2}, \lambda_{3}\right\}
$$

Suppose ( $\sigma i$, vi), $(i=1,2,3)$ are eigenvalue and normalized eigenvectors of $Q \Gamma$. Rearrange the order of ( $\sigma i$, vi), $(i=1,2,3)$ such that $\sigma 1 * \sigma 2>0$ and $|\sigma 1|>|\sigma 2|$. Then $\lambda i=\sigma i(i=1,2,3)$.

Let $P=[e 1, e 2, e 3]$. If v3 $*[0,0,1] T>0$, then $e 3=\mathrm{v} 3$, or else $e 3=-\mathrm{v} 3 . e 2=\mathrm{v} 2, e 1=e 2 \times e 3$.

In $O C-X^{\prime} Y^{\prime} Z^{\prime}$, there are two planes that their intersection with elliptic cone $\Gamma$ are circles and their radius are $R$,

$$
\left\{\begin{array}{l}
\left(x_{i}^{\prime}, y_{i}^{\prime}, z_{i}^{\prime}\right)=\left( \pm R \sqrt{\frac{\left|\lambda_{3}\right|\left(\left|\lambda_{1}\right|-\left|\lambda_{2}\right|\right)}{\left|\lambda_{1}\right|\left(\left|\lambda_{1}\right|-\left|\lambda_{3}\right|\right.}}, 0, R \sqrt{\frac{\left|\lambda_{1}\right|\left(\left|\lambda_{2}\right|+\left|\lambda_{3}\right|\right)}{\left|\lambda_{3}\right|\left(\left|\lambda_{1}\right|+\left|\lambda_{3}\right|\right)}}\right) \\
\left(n z_{x i}^{\prime}, n z_{y i}^{\prime}, n z_{z i}^{\prime}\right)=\left( \pm \sqrt{\frac{\left|\lambda_{1}\right|-\left|\lambda_{2}\right|}{\left|\lambda_{1}\right|+\left|\lambda_{3}\right|}}, 0,-\sqrt{\frac{\left|\lambda_{2}\right|+\left|\lambda_{3}\right|}{\left|\lambda_{1}\right|+\left|\lambda_{3}\right|}}\right)
\end{array}(i=1,2)\right.
$$

where $\left(x i^{\prime}, y i^{\prime}, z i^{\prime}\right)(i=1,2)$ are the center positions of the sectional circles in $O c-X^{\prime} Y^{\prime} Z^{\prime}$, and (nzxi', nzyi', nzzi' $)(i=1,2)$ are the two normal directions of the section planes. 
By transforming the circle positions and normal directions into camera reference frame $\{C\}$, the circle position and direction information can be got:

$$
\left\{\begin{array}{l}
\left(O_{B_{-} x i}^{\{C\}}, O_{B_{-} y i}^{\{C\}}, O_{B_{-} z i}^{\{C\}}\right)^{T}=P\left(x_{o i}^{\prime}, y_{o i}^{\prime}, z_{o i}^{\prime}\right)^{T} \\
\left(\vec{Z}_{B_{-} x i}^{\{C\}}, \vec{Z}_{B_{-} y i}^{\{C\}}, \vec{Z}_{B_{-} z i}^{\{C\}}\right)^{T}=P\left(n_{i x}^{\prime}, n_{i y}^{\prime}, n_{i z}^{\prime}\right)^{T}
\end{array}\right.
$$

2.2 False Solution Exclusion and Roll Angle Recovery via Single Line Projection

A. Determination of normal of plane $\pi 1$

From Fig. $1, \pi 1$ is a plane determined by $\mathrm{OC}$ and 1 . Since $\mathrm{K}$ is the intrinsic parameter matrix of camera and suppose $\{\mathrm{C}\}$ to be world reference frame, then the projection matrix of the camera is,

$$
\mathbf{M}=\left(\begin{array}{ll}
\mathbf{K} & 0
\end{array}\right)\left(\begin{array}{cc}
I_{3 \times 3} & \mathbf{0}_{3 \times 1} \\
\mathbf{0}_{1 \times 3} & 1
\end{array}\right)
$$

The homogenous coordinates of plane $\pi 1$ is,

$$
\hat{\boldsymbol{\pi}}_{1}=\left[\begin{array}{llll}
\pi_{1 x} & \pi_{1 y} & \pi_{1 z} & \pi_{1 w}
\end{array}\right]^{T}=\mathbf{M}^{T} \hat{l}
$$

Let the normal direction of $\pi 1$ be $N \pi 1=(\pi 1 x, \pi 1 y, \pi 1 z)$. Keep the angle between $N \pi 1$ and $O c X c$ acute angle, i.e, $\pi 1 x>0$; or else reverse the direction of $N \pi 1$.

B. Verification to exclude the false pose of circle

From Fig. $1, N \pi 1 \perp L$. Since $L \| \pi 2$, then $Z b \perp L$. Therefore,

$$
\vec{L}=\vec{Z}_{B} \times \vec{N}_{\pi_{1}}
$$

Since the angle between $\operatorname{ObZb}\{C\}$ and $O c Z c\{C\}$ is acute, as well as the angle between $N \pi 1$ and $O c X c\{C\}$, and $O b Y b=L$; therefore, $O b X b=O b Y b \times O b Z b$. Considering Eq.(6), we get the full pose the space object,

$$
\left\{\begin{array}{c}
\left(O_{B_{-} x i}^{\{C\}}, O_{B_{-} y i}^{\{C\}}, O_{B_{-} z i}^{\{C\}}\right)^{T}=P\left(x_{o i}^{\prime}, y_{o i}^{\prime}, z_{o i}^{\prime}\right)^{T} \\
\left(\vec{Z}_{B_{-} x i}^{\{C\}}, \vec{Z}_{B_{-} y i}^{\{C\}}, \vec{Z}_{B_{-} z i}^{\{C\}}=P\left(n_{i x}^{\prime}, n_{i y}^{\prime}, n_{i z}^{\prime}\right)^{T} \quad(i=1,2)\right. \\
\vec{Y}_{B_{-} i}^{\{C\}}=\vec{Z}_{B_{-} i} \times \vec{N}_{\pi_{1}} \\
\vec{X}_{B_{-} i}^{\{C\}}=\vec{Y}_{B_{-} i}^{\{C\}} \times \vec{Z}_{B_{-} i}
\end{array}\right.
$$

For any pose in Eq.(8), we can get the transformation from reference frame $\{B\}$ to $\{C\}$,

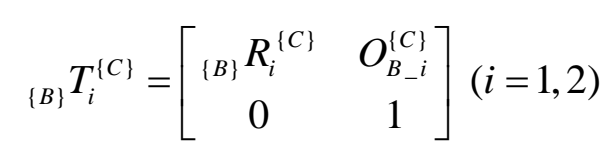

Where,

$$
{ }_{\{B\}} R_{i}^{\{C\}}=\left[\begin{array}{lll}
\vec{X}_{B_{-} i}^{\{C\}} & \vec{Y}_{B_{-} i}^{\{C\}} & \vec{Z}_{B_{-} i}^{\{C\}}
\end{array}\right]^{T}
$$

For any point $P$ on $L$, we can transform its position from reference frame $\{B\}$ to $\{C\}$,

$$
\left[\begin{array}{c}
P_{i}^{\{C\}} \\
1
\end{array}\right]={ }_{\{B\}} T_{i}^{\{C\}}\left[\begin{array}{c}
P^{\{B\}} \\
1
\end{array}\right](i=1,2)
$$

Then, its image $\mathrm{p}$ in I is,

$$
\lambda \hat{p}_{i}=\mathbf{K} P_{i}^{\{C\}}(i=1,2)
$$

Since $\mathrm{P} \in \mathrm{L}$, according invariance in projection transformation, there must be $\mathrm{p} \in \mathrm{l}$.

$$
\hat{l} \cdot \hat{p}^{T}=0
$$

Substitute Eq.(10) into Eq.(11), then only the pose which exactly satisfied Eq.(11) is the correct solution. Note that we need not to extract $\mathrm{p}$ in the image. We just get $\mathrm{p}$ by re-project $\mathrm{P}$ into the image with Eq.(10). 


\section{Experiments}

References are cited in the text just by square brackets [1]. (If square brackets are not available, slashes may be used instead, e.g. /2/.) Two or more references at a time may be put in one set of brackets $[3,4]$. The references are to be numbered in the order in which they are cited in the text and are to be listed at the end of the contribution under a heading References, see our example below.

In order to verify the proposed algorithm, physical experiment are carried out.

Physics experiments are mainly used to verify the feasibility of the algorithm in the real environment, and the accuracy of the algorithm is calculated under the real noise.

In order to verify the feasibility of this method in real environment, the experimental environment is set up:

1) The radius of the space circle is $75 \mathrm{~mm}$

2) The coordinates of a point on the space line are $(0,75,0)$, unit is $\mathrm{mm}$.

3) Experimental camera is Grasshopper3, image size is $1920 \times 1440$, principal point is (893726), focal ratios is $(1872,1868)$.

Because the real experiment can't get the theoretical position, but the camera's movement can be precisely controlled, so the precise motion control camera to simulate the movement of space objects.

Object reference position is $(1.94,-102.97,961.75)$.

Normal vector direction is $(-0.35,0.89,0.25)$.

Rotation angle reference direction is $(0.82,0.43,-0.35)$.

In the experiment, the camera move a certain distance along a certain direction. Then calculate the position and posture after the motion and compare with the results before motion. When the camera moves in a certain direction, the difference of position and posture should be close to zero.

Physical experiment results are shown in table 1. The first line represents a reference result, including a reference position, a reference vector, and a reference rotation angle. The first column represents the moving distance of the camera. The middle one is the position and posture results after the camera moves. The last column indicates the difference between current result with the reference result, including position, normal vector and rotation angle.

Table 1 Experiment Data

\begin{tabular}{ccl}
\hline Camera & $(47.24,-68.85,1011.80)$ & Contrast \\
movement & $(-0.35,0.89,0.25)$ & \\
distance & $(0.82,0.43,-0.35)$ & \\
\hline 91.54 & $(56.82,-59.13,1004.63)$ & 90.81 \\
& $(-0.39,0.87,0.26)$ & 2.74 \\
112.92 & $(0.78,0.47,-0.39)$ & 4.30 \\
& $(67.93,50.87,1026.84)$ & 116.09 \\
134.31 & $(-0.40,0.88,0.23)$ & 3.28 \\
& $(78.27,-44.02,1035,-0.38)$ & 3.14 \\
157.30 & $(-0.35,0.89,0.25)$ & 130.89 \\
& $(0.81,0.43,-0.38)$ & 0.57 \\
& $(88.69,-36.94,1056.32)$ & 1.80 \\
201.49 & $(-0.35,0.89,0.25)$ & 154.62 \\
& $(0.82,0.43,-0.35)$ & 0.42 \\
& $(110.95,-21.50,1087.77)$ & 0.29 \\
224.48 & $(-0.33,0.91,0.21)$ & 196.07 \\
& $(0.84,0.39,-0.35)$ & 2.39 \\
& $(123.26,-13.05,1107.07)$ & 2.57 \\
& $(-0.32,0.92,0.20)$ & 220.37 \\
& $(0.86,0.37,-0.34)$ & 4.41 \\
\end{tabular}

Analysis of the data in Table 1 can be found, when the camera moves along a certain direction as 91.54,112.92,134.31,157.30,201.49,224.48, the change of the center position are 
90.81,116.09,130.89,154.62,196.07,220.37. The normal vector and rotation angle are both small.

Considering the imaging error and the error in the real test, we can think the method is very good to solve the problem of the position and posture measurement.

\section{Summary}

In this paper, we use the circle and the linear structure of the non-cooperative target aircraft as the basis for the measurement of the position and posture. Due to only use a circle and a parallel line, it can reduce the difficulty of feature selection and recognition, and improve the reliability and robustness of the method.

Compared with the prior art, the advantage is that:

(1) The characteristics of the circle and the line are more stable.

(2) Less difficult feature selection because only need to use a circle and a straight line.

(3) The whole position and posture of the non-cooperative target aircraft can be determined.

\section{References}

[1]. Pan H, Huang J Y, Qin S Y. Relative Pose Estimation under Monocular Vision in Rendezvous and Docking[J]. Applied Mechanics \& Materials, 2013, 433-435:799-805.

[2]. Zhang S J, Cao X B, Hui L I. Closed-form method for relative posture and position parameters of RVD spacecrafts based monocular vision[J]. Optical Technique, 2005.

[3]. Tafazoli M. A study of on-orbit spacecraft failures[J]. Acta Astronautica, 2009, 64(64):195-205.

[4]. Benninghoff H, Boge T, Rems F. Autonomous Navigation for On-Orbit Servicing[J]. KI Künstliche Intelligenz, 2014, 28(2):77-83.

[5]. Yongzhi Z J W J W, Zejian Z, Jie A W. High-Precision Navigation Approach of High-Orbit Spacecraft Based on Retransmission Communication Satellites[J]. Journal of Navigation, 2012, 65(2):351-362.

[6]. Shan G, Han Yanhua|. A Vision-Based Relative Position and Posture Determination Algorithm for RVD between Spacecraft[J]. Aerospace Control, 2011, 29(1):31-36.

[7]. Nair P S, Saunders A T. Hough transform based ellipse detection algorithm[J]. Pattern Recognition Letters, 1996, 17(7):777-784.

[8]. Kim E, Haseyama M, Kitajima H. Fast and Robust Ellipse Extraction from Complicated Images[C]// Proceedings of the IEEE International Conference on Information Technology and Applications. 2010.

[9]. Zhang H X, Zhao J G, Jian-Feng L I. A Measure Method of Relative Position and Posture Between Spacecrafts Based on Target Feature[J]. Journal of Institute of Command \& Technology, 2004.

[10]. Shiu Y C, Ahmad S. 3D location of circular and spherical features by monocular model-based vision[C]// IEEE International Conference on Systems, Man and Cybernetics, 1989. Conference Proceedings. 2010:576-581 vol.2.

[11]. Safaeerad R, Tchoukanov I, Smith K C, et al. Three-dimensional location estimation of circular features for machine vision[J]. IEEE Transactions on Robotics \& Automation, 1992, 8(5):624-640.

[12]. 苗锡奎, 朱枫, 丁庆海, 等. 基于星箭对接环部件的飞行器单目视觉位姿测量方法 $[\mathrm{J}]$. 光学 学报, 2013(4):115-123. 